\title{
Molecular characterization of pearl millet [Pennisetum glaucum (L.) R. Br] inbreds using microsatellite markers
}

\author{
Mamta Nehra ${ }^{1 *}$, Mukesh Kumar ${ }^{2}$, Dev Vart ${ }^{3}$, Jyoti Kaushik ${ }^{4}$ and Rajesh Kumar Sharma ${ }^{5}$ \\ Department of Genetics and Plant Breeding, CCS Haryana Agricultural University, Hisar-125004 (Haryana), INDIA \\ *Corresponding author. E-mail: mamtanehra089@gmail.com
}

Received: June 30, 2016; Revised received: December 7, 2016; Accepted: February 3, 2017

Abstract: Studies on genetic diversity in Pennisetum germplasm are the promising opportunities for the use of undomesticated materials for improving pearl millet varieties. DNA based markers have now emerged as a potential genomic tool for estimation of genetic diversity among various cultivars and varietal identification. In present study, genetic diversity among 49 stay green inbreds of pearl millet was studied using simple sequence repeats (SSRs). Twenty nine polymorphic SSR primers, identified after initial screening of 70 , were used to study diversity among these lines. A total of 108 alleles were amplified, collectively yielding unique SSR profiles for all the 49 inbreds. The average number of SSR alleles per locus was 3.72, with a range from 2 to 13 . Polymorphic information content (PIC) values of various SSR loci across all the 49 inbreds ranged from 0.14 to 0.87 with an average of 0.51 per locus. This indicated sufficient diversity among the 49 pearl millet inbreds and total 5 out of 29 polymorphic SSR loci, namely Xpsmp2070, Xpsmp2001, Xpsmp2008, Xpsmp2066, Xpsmp2072 revealed PIC values above 0.70, can be considered highly useful for differentiation of pearl millet inbred lines. The lowest PIC value (0.47) for linkage group 7 showed comparatively conserved nature of this linkage group A dendrogram obtained using WARD's minimum variance method further delineates 49 inbreds into 8 major clusters, and the clustering pattern corroborated with their pedigree and characteristics traits.

Keywords: Pearl millet, Genetic diversity, PIC, SSR Marker, Dendrogram

\section{INTRODUCTION}

Pearl millet [Pennisetum glauccum (L.) R. Br.] is a summer annual crop originating from Africa, from where it was introduced into other regions of the world with diverse agro-climatic conditions that is, from the hot area of Africa (the tropical zone) to the hot area of temperate zones. Therefore a large number of diversity is found within and among pearl millet cultivars. Due to its highly out-crossing breeding behavior, pearl millet was originated from several independent domestication events and wide range of stressful environmental conditions, in which it had been traditionally cultivated. Pearl millet exhibits a tremendous amount of diversity at both phenotypic and genotypic levels (Liu et al., 1994). Estimation of genetic diversity and identification of superior genotypes are some of the prime objectives of any crop improvement programmes. Highly diverse genotypes or accessions can be utilized as parents in hybridization programmes to produce superior varieties/hybrids. Therefore there is a need to evaluate available genotypes for their genetic diversity (Sumathi and Vinodhana, 2014).

Pearl millet is a $\mathrm{C}_{4}$ monocot species belonging to the Poaceae family and has a relatively small diploid genome $(2 \mathrm{n}=2 \mathrm{x}=14)$ with a DNA content of $2.36 \mathrm{pg}$. It is a highly cross-pollinated crop with protogynous flowering nature which fulfils one of the essential biological requirements for hybrid development. Pearl millet is one of the major cereals grown, primarily for grain production, on more than $29 \mathrm{~m}$ ha in the arid and semi-arid tropical regions of Africa and Asia (Kannan et al., 2014). India is the largest producer of pearl millet in Asia and occupied an area of $7.95 \mathrm{~m}$ ha with production of $8.79 \mathrm{mt}$ and productivity $1106 \mathrm{~kg} / \mathrm{ha}$ (Anonymous, 2014). Pearl millet is drought tolerant and 'high energy' coarse cereal with high starch (70\%) in dry grain, protein (10-12\%) and 5-7\% fat. Adaptability to diverse environmental conditions has made it a preferred crop in areas where other crops like maize or wheat would not survive. It is primarily cultivated for grain, but is also a valuable source of fodder.. The energy density of pearl millet is relatively high, arising from its higher oil content relative to maize, wheat or sorghum (Hill and Hanna, 1990). Pearl millet contains 27 to $32 \%$ more protein than maize, higher concentrations of essential amino acids, twice the ether extract, and higher gross energy than maize (Ejeta et al., 1987). Genetic diversity studies in pearl millet germplasm offer possibilities for their use in improving pearl millet open-pollinated varieties and hybrids. Molecular markers are helpful in diversity analysis as they are not influenced by environment and provide more accurate results. DNA based molecular markers have been suc- 
cessfully employed in quantification of genetic diversity, genotype identification, delineation and marker assisted selection. These have been effectively utilized for the assessment of genetic diversity as compared to the morphological or biochemical due to their abundance, high level of polymorphism, reproducibility and being independent of the environment. Molecular markers namely RFLP, RAPD, ISSR and SSRs are mostly used, among these microsatellite or SSRs has become the marker of choice for many applications due to their abundance, high level of repeats number, polymorphisms, manifested as the occurrence of the large number of alleles per locus, and co-dominant inheritance has facilitated their extensive use in genome mapping, phylogenetic inference and population genetics. Simple sequence repeats (SSRs) have been found as more reliable because they are highly polymorphic, informative and co-dominant markers which are often broadly applicable since loci are frequently conserved between related species and sometimes genera (Chakauya and Tongoona, 2008). The integration of genomic technologies in pearl millet breeding will improve the efficiency of breeding programs in the development of better cultivars and reduce the time required for cultivar development.

Thus, in the present investigation, SSR markers were used to characterize the pattern of diversity among pearl millet inbreds to identify better inbreds for the development of commercial hybrids in pearl millet.

\section{MATERIALS AND METHODS}

Plant materials: Forty nine pearl millet stay green inbreds were selected on the basis of performance and sown in a randomized block design (RBD) with three replications in the field of Bajra section, Genetics and Plant Breeding Department, CCS HAU, Hisar, Haryana (Table 1).

A set of 70 highly polymorphic SSR markers covering different genomic locations were selected for this study (Table 2). The genomic locations for most of the markers were derived from the pearl millet genome map.

Genomic DNA extraction: Genomic DNA was isolated from the young leaves of pearl millet by CTAB (Cetyltrimethylammonium bromide) extraction method as given by Murray and Thompson (1980) and modified by Saghai-Maroof et al. (1984) and Xu et al. (1994). DNA was subsequently dissolved in appropriate volume of T.E. buffer and samples were stored at $20^{\circ} \mathrm{C}$ for further use. DNA concentration was determined using the UV spectro-photometer at 260 and $280 \mathrm{~nm}$. PCR amplifications were performed using applied biosystems thermal cycler. The PCR reaction was carried out in a reaction volume of $20 \mu \mathrm{l}$ containing $10 \mathrm{X}$ PCR buffer, $100 \mu \mathrm{M}$ each of dNTPs, $0.4 \mu \mathrm{M}$ of each primer, 1 unit Taq DNA polymerase and $50 \mathrm{ng}$ template DNA. PCR amplification was performed with initial denaturation at $94^{\circ} \mathrm{C}$ for 5 min followed by 10 cycles of denaturation at $94^{\circ} \mathrm{C}$ for $25 \mathrm{~s}$, annealing at $64^{\circ} \mathrm{C}-54^{0} \mathrm{C}$ (touch-down cycles) for $20 \mathrm{~s}$, and extension at $72^{\circ} \mathrm{C}$ for $30 \mathrm{~s}$, followed by 40 cycles of denaturation at $94^{\circ} \mathrm{C}$ for $25 \mathrm{~s}$, annealing at $56^{\circ} \mathrm{C}$ for $20 \mathrm{~s}$ and exten-

Table 1. List of pearl millet stay green inbred lines used for conduct experiment.

\begin{tabular}{|c|c|c|}
\hline $\begin{array}{l}\text { S. } \\
\text { No. }\end{array}$ & $\begin{array}{l}\text { Name of inbred } \\
\text { line }\end{array}$ & Pedigree \\
\hline 1. & HSGR-01 & SPF 2 98-2 \\
\hline 2. & HSGR-02 & H 90/4-5 X 77/ 29-2 \\
\hline 3. & HSGR-03 & HTP 92/5 \\
\hline 4. & HSGR-04 & $\begin{array}{l}\text { (ICMB } 92333 \text { X EEBC CI-I)-5 } \\
\text {-B-B }\end{array}$ \\
\hline 5. & HSGR-05 & $\begin{array}{l}\text { K-560-2 X(J 834-7 X } 700544 \\
-7-2-1)\end{array}$ \\
\hline 6. & HSGR-06 & HTP 3/14 \\
\hline 7. & HSGR-07 & VCF $6862 / 98-1$ \\
\hline 8. & HSGR-08 & AC-04 /6 \\
\hline 9. & HSGR-09 & $1210 / 1$ \\
\hline 10. & HSGR-10 & H 94 / 61-2 \\
\hline 11. & HSGR-11 & JBV 3 S1 - 44-3 -B -4 -B \\
\hline 12. & HSGR-12 & HTP - 07-26 \\
\hline 13. & HSGR-13 & НTP 07-44 \\
\hline 14. & HSGR-14 & $96 \mathrm{AC}-99$ \\
\hline 15. & HSGR-15 & $\begin{array}{l}\text { HMP - } 0810 \text { ( ICMA } 01222 \text { X } \\
\text { ICMP 451) }\end{array}$ \\
\hline 16. & HSGR-16 & SPF $298-2$ \\
\hline 17. & HSGR-17 & VCF 41864 \\
\hline 18. & HSGR-18 & НTP $92 / 110$ \\
\hline 19. & HSGR-19 & SGP $-10-110$ \\
\hline 20. & HSGR-20 & HTP -10 -137 \\
\hline 21. & HSGR-21 & PT - 1-10 -1038 \\
\hline 22. & HSGR-22 & PT-1-10 - 1043 \\
\hline 23. & HSGR-23 & TCF $3-10-3-2$ \\
\hline 24. & HSGR-24 & TCF $3-10-28-5$ \\
\hline 25. & HSGR-25 & PT -1 - $10-1099$ \\
\hline 26. & HSGR-26 & DMRC - 09 / 11-81 -2 \\
\hline 27. & HSGR-27 & High Fe JBT / $12-122$ \\
\hline 28. & HSGR-28 & TPRT / 12- 119 \\
\hline 29. & HSGR-29 & TCPTA / $12-128$ \\
\hline 30. & HSGR-30 & 110041 \\
\hline 31. & HSGR-31 & HPT - $2-12-7$ \\
\hline 32. & HSGR-32 & $99 \mathrm{HS}-22$ \\
\hline 33. & HSGR-33 & 2305 \\
\hline 34. & HSGR-34 & MIR 97041 \\
\hline 35. & HSGR-35 & G $73-107-05 \mathrm{~K}-1$ \\
\hline 36. & HSGR-36 & AC-04 /13 \\
\hline 37. & HSGR-37 & $99 \mathrm{HS}-23$ \\
\hline 38. & HSGR-38 & 99HS -145 \\
\hline 39. & HSGR-39 & 98 Raj 4 \\
\hline 40. & HSGR-40 & $99 \mathrm{ABL}-5$ \\
\hline 41. & HSGR-41 & $\begin{array}{l}\{\text { ICMB } 91777 \text { X ( } 91777 B \text { X } \\
\text { HHVBC })\}-6-B\end{array}$ \\
\hline 42. & HSGR-42 & $\mathrm{HBL}-34$ \\
\hline 43. & HSGR-43 & 1660(M.T.) \\
\hline 44. & HSGR-44 & HF IT - $1-129$ \\
\hline 45. & H 77 / 833 -2-202 & \\
\hline 46. & H 77 / $833-2$ & \\
\hline 47. & H77 / $29-2$ & \\
\hline 48. & HBL - 11 & \\
\hline 49. & ICMR 01004 & \\
\hline
\end{tabular}


Mamta Nehra et al. / J. Appl. \& Nat. Sci. 9 (1): 357 - 363 (2017)

Table 2. List of primers and their sequences used in the present study.

\begin{tabular}{|c|c|c|c|c|}
\hline $\begin{array}{c}\text { S. } \\
\text { No. }\end{array}$ & Linkage group & Primer name & Sequence (forward) & Sequence (reverse) \\
\hline 1 & LG1 & Xctm12 & GTTGCAAGCAGGAGTAGATCGA & CGCTCTGTAGGTTGAACTCCTT \\
\hline 2 & LG1 & Xctm27 & GTTGCAAGCAGGAGTAGATCGA & CGCTCTGTAGGTTGAACTCCTT \\
\hline 3 & LG1 & Xicmp3017 & CACCAAACAGCATCAAGCAG & AGGTAGCCGAGGAAGGTGAG \\
\hline 4 & LG1 & Xicmp3032 & AGGTAGCCGAGGAAGGTGAG & CAACAGCATCAAGCAGGAGA \\
\hline 5 & LG1 & Xicmp3080 & CAAACAGCATCAAGCAGGAG & GCGTAGACGGCGTAGATGAT \\
\hline 6 & LG1 & Xipes0009 & TTGATCGATCGTCTACGGTT & TATACTCACTCACGGCAGCG \\
\hline 7 & LG1 & Xipes0042 & GATAGAAGCAGATGGGCCTG & CTCGTCATCATTCTCGCCAC \\
\hline 8 & LG1 & Xipes0079 & GTTGGACAGGCGAACGATAC & AGCTCTCCTGCATTTTCGTG \\
\hline 9 & LG1 & Xipes0098 & ATCAAGCTTCATACCCCTGC & CATCTTCTTCATCATCTTTCGC \\
\hline 10 & LG1 & Xipes0126 & CCAGCAGGGAAGTCTTTCAC & AAAGGCGCTTGCTGATTTT \\
\hline 11 & LG1 & Xipes0139 & GTGTATGGTATGCGTGTCCG & GCATTGTGATCGAATAAACTACTG \\
\hline 12 & LG1 & Xipes0146 & CATCAGAATACGGACGCCTT & CATCAGCTTTGGAGTCAGCA \\
\hline 13 & LG1 & Xipes0197 & GTGTTCTTCCGAATCCGTGT & CGCTTTGCATTGAACACAGT \\
\hline 14 & LG1 & Xipes0203 & CCCTCGAAGAGATCGAAGTG & CTGAAACAACAGCCTGCAAA \\
\hline 15 & LG1 & Xipes0216 & AAAGGCAGCAGATCCCTACA & TCTTTCGTTGCATCTGTTCG \\
\hline 16 & LG1 & Xpsmp2006 & GACTTATAGTCACTGGGAAAGCTC & GCTTTAATAACTTTGTGCGTATT \\
\hline 17 & LG1 & Xpsmp2030 & ACCAGAGCTTGGAAATCAGCAC & CATAATGCTTCAAATCTGCCACAC \\
\hline 18 & LG1 & Xpsmp2069 & CCCATCTGAAATCTGGCTGAGAA & CCGTGTTCGTACAAGGTTTTGC \\
\hline 19 & LG1 & Xpsmp2080 & CAGAATCCCCACATCTGCAT & TGCAACTGAGCGAAGATCAA \\
\hline 20 & LG1 & Xpsmp2090 & AGCAGCCCAGTAATACCTCAGCTC & AGCCCTAGCGCACAACACAAACTC \\
\hline 21 & LG1 & Xpsmp2232 & TGTTGTTGGGAGAGGGTATGAG & CTCTCGCCATTCTTCAAGTTCA \\
\hline 22 & LG1 & Xpsmp2273 & AACCCCACCAGTAAGTTGTGCTGC & GATGACGACAAGACCTTCTCTCC \\
\hline 23 & LG2 & Xctm03 & GTCCATCGTCGCCGACGAA & GGATTTGCTAGTTGTGGGCT \\
\hline 24 & LG2 & Xctm21 & ATGCCTCCCACCCCACGTCG & CGTCGCACTAGCCACAGTCA \\
\hline 25 & LG2 & Xpsmp2050 & ATCAAACGGCATCAGACAAC & GGATCTCTTAGTGTGGTGGAGAGC \\
\hline 26 & LG2 & Xpsmp2059 & GGGGAGATGAGAAAACACAATCAC & TCGAGAGAGGAACCTGATCCTAA \\
\hline 27 & LG2 & Xpsmp2066 & ATATTAGAGCATTGCATCGC & GCATAGCAGCATACAGCAGCAAAA \\
\hline 28 & LG2 & Xpsmp2068 & CAATAACCAAACAAGCAGGCAG & СТТСАСТСССАСССТТТСТААТТС \\
\hline 29 & LG2 & Xpsmp2072 & GAAATCTACACAAGGGTCTCCA & GTACGGCAGAATGACATCTGAA \\
\hline 30 & LG2 & Xpsmp2077 & GCCAATATTATTCCCAAGTGAACA & CTCTTGGTTGCATATCTTTCTTTT \\
\hline 31 & LG2 & Xpsmp2088 & AAGAAGCCACCAGCACAAAA & TGCATGAAAGTAGAGGATGGTAAA \\
\hline 32 & LG2 & Xpsmp2089 & TTCGCCGCTGCTACATACTT & TGTGCATGTTGCTGGTCATT \\
\hline 33 & LG2 & Xpsmp2237 & TGGCCTTGGCCTTTCCACGCTT & CAATCAGTCCGTAGTCCACACCCCA \\
\hline 34 & LG3 & Xctm10 & GAGGCAAAAGTGGAAGACAG & TTGATTCCCGGTTCTATCGA \\
\hline 35 & LG3 & Xpsmp2056 & ACCTGTAGCTTCAAAATTCAAAAA & AATTCAGTGTGATTTCGATGGTTGC \\
\hline 36 & LG3 & Xpsmp2070 & ACAGAAAAAGAGAGGCACAGGAGA & GCCACTCGATGGAAATGTGAAA \\
\hline 37 & LG3 & Xpsmp2071 & TTGCAGTCCCACGAATTATTTG & CTTTGAATTTATAATCCTCATACT \\
\hline 38 & LG3 & Xpsmp2214 & CGCACAGTACGTGTGAGTGAAG & GATTGAGCAGCAAAAACCAGC \\
\hline 39 & LG4 & Xipes0066 & CAACATGTCAAGGAAGTAAAATTGA & GCCTCTTGATACCCAAGATCA \\
\hline 40 & LG4 & Xipes0114 & CGTTGTGTTGAATAATGTCGTACC & CAATAACCAAACGACGGACA \\
\hline 41 & LG4 & Xipes0186 & AGCATATGGCATCCTTTTCG & TTTCAGGCTTGGATTCAATGT \\
\hline 42 & LG4 & Xipes0208 & CGAAGGAGGAGTACGACGAG & TCCACAAGGTGACCTCACTG \\
\hline 43 & LG4 & Xipes0225 & CAAACCTCAAGCTAGGCGAC & CATGCATACACCAGTGCCAT \\
\hline 44 & LG4 & Xpsmp2008 & GATCATGTTGTCATGAATCACC & ACACTACACCTACATACGCTCC \\
\hline 45 & LG4 & Xpsmp2076 & GGAATAGTATATTGGCAAAATGTG & ATACTACACACTGTAAGCATTGTC \\
\hline 46 & LG4 & Xpsmp2081 & CTGTGCTGTCATTGTTACCA & TCAGATCACCTATTACTTTCCCT \\
\hline 47 & LG4 & Xpsmp2084 & AATCTAGTGATCTAGTGTGCTTCC & GGTTAGTTTGTTTGAGGCAAATGC \\
\hline 48 & LG4 & Xpsmp2085 & GCACATCATCTCTATAGTATGCAG & GCATCCGTCATCAGGAAATAA \\
\hline 49 & LG4 & Xpsmp2086 & CGCTTGTTTTCCTTTCTTGCTGTT & ССTTCTCAGATCCTGTGCTTTCTT \\
\hline 50 & LG4 & Xpsmp3029 & ATCGATCTGTTCCACCCAGT & GGACTGGTACTGCTGCTGCT \\
\hline 51 & LG5 & Xpsmp2001 & CATGAAGCCAATTAGGTCTC & ACCATCTGACTTGTTCTTATCC \\
\hline 52 & LG5 & Xpsmp2064 & ACCGAATTAAAGTCATGGATCG & TTGATTCTTCTGACACAAATGAG \\
\hline 53 & LG5 & Xpsmp2078 & CATGCCCATGACAGTATCTTAAT & ACTGTTCGGTTCCAAAATACTT \\
\hline 54 & LG6 & Xicmp3086 & ACCAAACGTCCAAACCAGAG & ATATCTCTTCGCTGCGGTGT \\
\hline 55 & LG6 & Xpsmp2018 & CGCAAGACATTTTAGTATCACC & ACAGTCATCCTCAGTCGTCC \\
\hline 56 & LG6 & Xpsmp2048 & TGAATTGGGAATAAAGGAGACC & ACGTGTGCCTGCTTTTAGTAAC \\
\hline 57 & LG6 & Xpsmp2270 & AACCAGAGAAGTACATGGCCCG & CGACGAACAAATTAAGGCTCTC \\
\hline 58 & LG7 & Xctm08 & GCTGCATCGGAGATAGGGAA & CTCAGC AAGCACGCTGCTCT \\
\hline 59 & LG7 & Xpsmp2013 & GTAАCCСАСТААСССТТАCC & GTCGCACAGAAAAAGAATAG \\
\hline 60 & LG7 & Xpsmp2019 & TGTGCCACAGCTTGTTCCTC & CAAGCAGCCAGTTCCTCATC \\
\hline 61 & LG7 & Xpsmp2027 & AGCAATCCGATAACAAGGAC & AGCTTTGGAAAAGGTGATCC \\
\hline 62 & LG7 & Xpsmp2033 & CTATACCATTGAATTGAAAGGTC & CAATCTTTAGCTTTTTCAAGAGAC \\
\hline 63 & LG7 & Xpsmp2040 & CATTACACGTTTCTTCAAACGC & TCTTCGGCCTAATAGCTCTAAC \\
\hline 64 & LG7 & Xpsmp2043 & TCATATTCTCCTGTCTAAAACGTC & ACAAATCGTACAAGTTCCACTC \\
\hline 65 & LG7 & Xpsmp2063 & GAGCACATGAAATAGGAAGCAG & AAGGTAGTTATAGTTAGCTTGATC \\
\hline 66 & LG7 & Xpsmp2074 & AGGACTGTAGGAGTGTGGACAACACA & CCAGACCTACCAGTGAATGAGA \\
\hline 67 & LG7 & Xpsmp2079 & AGCCGAAGGCTAATCAACAA & GTGGTCAGCAGCAGATGTAA \\
\hline 68 & LG7 & Xpsmp2087 & GGAACAGACTCCATACCTGAAA & TACCTGCCTGTGCTGTTAGT \\
\hline 69 & LG7 & Xpsmp2203 & GAACTTGATGAGTGCCACTAGC & TTGTGTAGGGAGCAACCTTGAT \\
\hline 70 & LG7 & Xpsmp2224 & GGCGAATTGGAATTCAGATTG & CGTAATCGTAGCGTCTCGTCTAA \\
\hline
\end{tabular}


sion at $72^{\circ} \mathrm{C}$ for $30 \mathrm{~s}$, followed by final extension at $72^{0} \mathrm{C}$ for $20 \mathrm{~min}$. Amplification products were separated on $2.5 \%$ agarose gel containing ethidium bromide and visualized under UV light using gel documentation system.

Molecular data analysis: To estimate genetic similarity/dissimilarities between different inbreds the data of banding pattern was used as input. The presence of band run on agarose gel was taken as one and absence of band was read as zero. The binary data was used to calculate similarity genetic distance using JMP 8.0 software, SAS Institute Inc., Carry, NC, 1989-2007. Dendrogram was constructed by using distance matrix by the unweighted pair group method using arithmetic averages (UPGMA) of JMP 8.0 Software. The genetic diversity of each microsatellite locus was assessed by calculating the frequency of the microsatellite alleles based on polymorphic information content (PIC). PIC is a measure of allele diversity at a locus and formula is given by

$\mathrm{PIC}=1-\sum_{\mathrm{j}=1}{ }^{\mathrm{n}} \sum \mathrm{P}_{\mathrm{ij}}^{2}$

Where, pij is the frequency of the $\mathrm{jth}$ allele for ith marker (Anderson et al., 1993). PIC is synonymous with the term "gene diversity" as described by Weir (1996). The PIC value ranging from ' 0 ' (Monomorphic) to ' 1 ' (highly discriminative with many alleles in equal frequency) is an indication of discriminative power of marker, not only for number

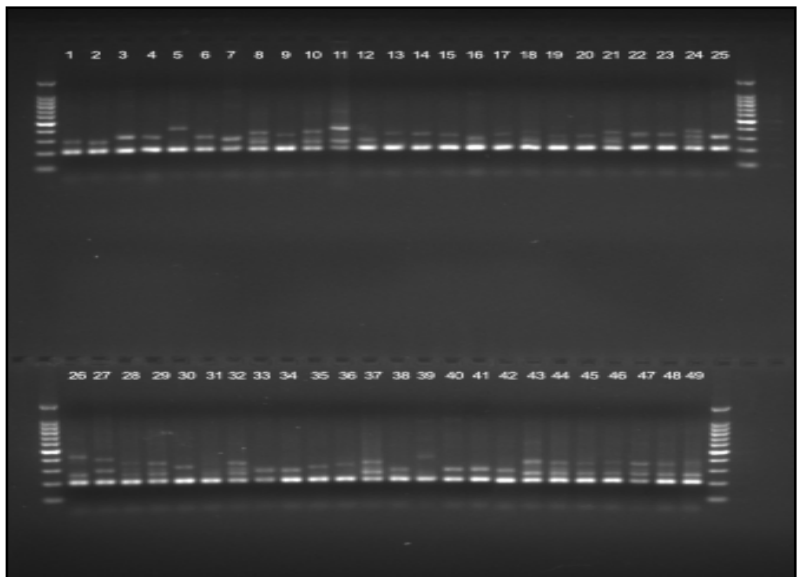

Fig. 1. Polymorphism in different forty nine inbreds of pearl millet by using primer Xpsmp2001.

of alleles at a locus but also for relative frequencies of those allele in the inbreds under study.

\section{RESULTS AND DISCUSSION}

Molecular marker analysis: The DNA was extracted from 49 inbreds of pearl millet and checked for quality and quantity. The spectrophotometer revealed the 260/280 ratio of about 1.7-1.9 which indicated the presence of good quality of genomic DNA. The electrophoretic separation of genomic DNA on $0.8 \%$ agarose gel revealed the presence of single intact band.

Table 3. List of SSR primers showing polymorphism.

\begin{tabular}{|c|c|c|c|c|c|c|}
\hline S.N. & SSR Locus & SSR Motif & $\begin{array}{l}\text { Number of } \\
\text { alleles }\end{array}$ & $\begin{array}{c}\text { Range of band sizes } \\
\text { (bp) }\end{array}$ & $\begin{array}{l}\text { Linkage } \\
\text { group }\end{array}$ & $\begin{array}{c}\text { PIC } \\
\text { values }\end{array}$ \\
\hline 1 & Xpsmp2001 & $(\mathrm{TC})_{8}(\mathrm{AC})_{47}$ & 6 & $200-450$ & LG5 & 0.72 \\
\hline 2 & Xpsmp2008 & $(\mathrm{TG})_{38}(\mathrm{CT})_{1}$ & 8 & $180-1000$ & LG4 & 0.85 \\
\hline 3 & Xpsmp2013 & $(\mathrm{GT})_{10}$ & 4 & $150-300$ & LG7 & 0.34 \\
\hline 4 & Xpsmp2019 & $(\mathrm{CA})_{38}$ & 3 & $200-300$ & LG7 & 0.61 \\
\hline 5 & Xpsmp2027 & $(\mathrm{GT})_{31}$ & 3 & $250-600$ & LG7 & 0.14 \\
\hline 6 & Xpsmp 2030 & $(\mathrm{CA})_{11}(\mathrm{GA})_{10}$ & 2 & $100-200$ & LG1 & 0.48 \\
\hline 7 & Xpsmp2043 & $(\mathrm{CA})_{13}(\mathrm{GA})_{6}$ & 2 & $200-700$ & LG7 & 0.45 \\
\hline 8 & Xpsmp2048 & $(\mathrm{AC})_{33}$ & 2 & $200-400$ & LG6 & 0.49 \\
\hline 9 & Xpsmp2063 & $(\mathrm{AC})_{22}(\mathrm{AT})_{5}$ & 3 & $100-500$ & LG7 & 0.59 \\
\hline 10 & Xpsmp2066 & $(\mathrm{CA})_{54}$ & 4 & $200-500$ & LG2 & 0.70 \\
\hline 11 & Xpsmp2069 & $(\mathrm{CA})_{19} \mathrm{CTCG}(\mathrm{CA})_{7}$ & 3 & $200-600$ & LG1 & 0.57 \\
\hline 12 & Xpsmp 2070 & $(\mathrm{CA})_{25}(\mathrm{TA})_{6}$ & 13 & $200-1200$ & LG3 & 0.87 \\
\hline 13 & Xpsmp 2072 & $(\mathrm{CA})_{24} \mathrm{TC}(\mathrm{TA})_{5}$ & 12 & $100-1000$ & LG2 & 0.82 \\
\hline 14 & Xpsmp2077 & $(\mathrm{CA})_{15}(\mathrm{TA})_{8}$ & 2 & $150-250$ & LG2 & 0.42 \\
\hline 15 & Xpsmp2078 & $(\mathrm{CA})_{42}$ & 3 & $100-250$ & LG5 & 0.61 \\
\hline 16 & Xpsmp2084 & $(\mathrm{CA})_{44}$ & 3 & $200-300$ & LG4 & 0.64 \\
\hline 17 & Xpsmp2085 & $(\mathrm{AC})_{11}$ & 2 & $150-200$ & LG4 & 0.35 \\
\hline 18 & Xpsmp2086 & $(\mathrm{CA})_{13}$ & 2 & $80-150$ & LG4 & 0.46 \\
\hline 19 & Xpsmp2088 & $(\mathrm{CA})_{24}$ & 4 & $100-700$ & LG2 & 0.61 \\
\hline 20 & Xpsmp2089 & $(\mathrm{AC})_{14}$ & 4 & $100-400$ & LG2 & 0.54 \\
\hline 21 & Xpsmp2203 & $(\mathrm{GT})_{18}$ & 2 & $300-400$ & LG7 & 0.46 \\
\hline 22 & Xpsmp 2237 & $(\mathrm{GT})_{8}$ & 2 & $200-300$ & LG2 & 0.30 \\
\hline 23 & Xpsmp 2273 & $(\mathrm{GA})_{12}$ & 5 & $150-600$ & LG1 & 0.61 \\
\hline 24 & Xctm10 & $(\mathrm{CT}) 22$ & 2 & $180-230$ & LG3 & 0.48 \\
\hline 25 & Xctm 21 & $(\mathrm{CT})_{24}$ & 4 & $150-500$ & LG2 & 0.51 \\
\hline 26 & Xipes0009 & $(\mathrm{CGTA})_{6}$ & 2 & $150-200$ & LG1 & 0.40 \\
\hline 27 & Xipes0042 & $(\mathrm{TGA})_{7}$ & 2 & $350-450$ & LG1 & 0.14 \\
\hline 28 & Xipes0098 & $(\mathrm{TGA})_{12}$ & 2 & $200-300$ & LG1 & 0.40 \\
\hline 29 & Xipes0146 & $(\mathrm{ATCTTC})_{6}$ & 2 & $100-200$ & LG1 & 0.49 \\
\hline
\end{tabular}




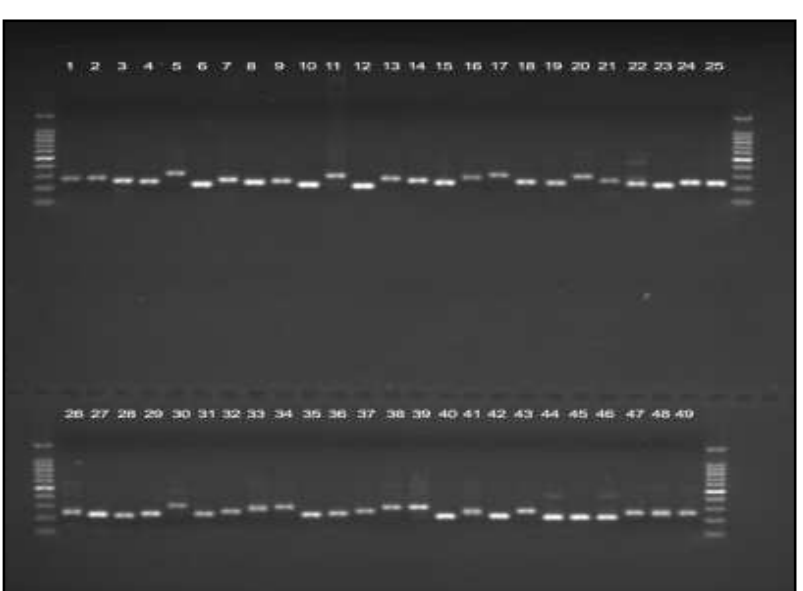

Fig. 2. Polymorphism in different forty nine inbreds of pearl millet by using primer Xpsmp2084.

The present study comprised of detailed assessment of the utility of SSR markers in effectively discriminating forty nine inbred lines. Total 29 pair polymorphic SSR primers were identified on the basis of initial screening of 70 primers, used for assessing genetic diversity among pearl millet inbreds. A total of 108 alleles were detected, collectively yielding unique SSR profiles for all the 49 inbreds. Results given in Table 3 revealed that the average number of SSR alleles per locus was 3.72 , with a range from 2 (Xpsmp2077, Xpsmp2203, Xpsmp2237, Xpsmp2086, Xpsmp2030, Xpsmp2085, Xpsmp2043, Xpsmp2048, Xctm10, Xipes0009, Xipes0098, Xipes0042, Xipes0146) to 13 (Xpsmp2070). Singh et al. (2013) identified total of 21 pair polymorphic SSR primers on the basis of initial screening of 60 , were used for assessing genetic diversity among the pearl millet cultivars. These primers amplified a total of 64 alleles, which varied from 2 to 6 per locus, with a mean of 3.0 per locus. The overall polymorphism among the cultivars was $92 \%$.

Based on the allele frequencies, PIC (Polymorphism Information Content) values for different SSR loci were estimated and found that value ranged from 0.14 (Xpsmp2027, Xipes0042) to 0.87 (Xpsmp2070) with an average of 0.51 per locus. Figure 1 and 2 shows a sample of polymorphic bands generated by primers Xpsmp2001 and Xpsmp2084 respectively. It is significant to note that 5 out of 29 SSR loci, namely Xpsmp2070, Xpsmp2001, Xpsmp2008, Xpsmp2066,

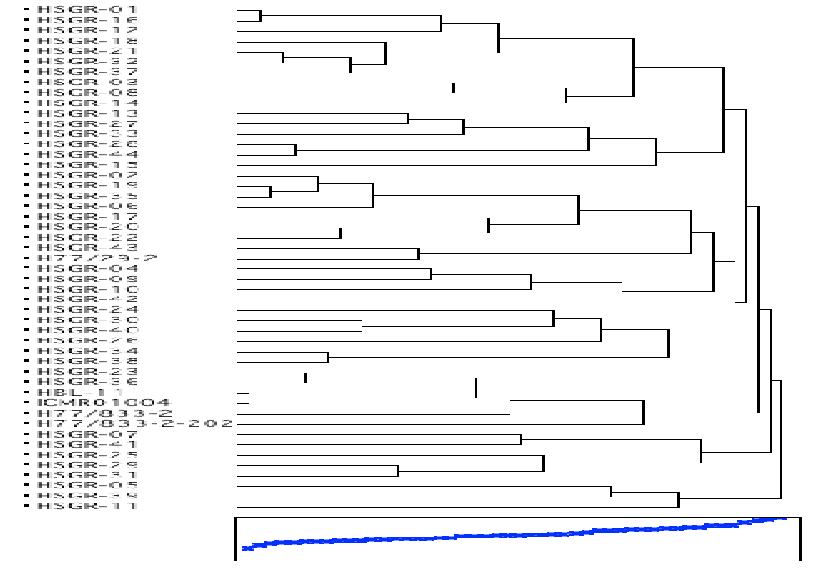

Fig. 3. Dendrogram showing the clustering pattern of forty nine inbreds of pearl millet on the basis of SSR marker consequences.

Xpsmp2072 revealed PIC values above 0.70, can be considered highly useful for differentiation of pearl millet inbred lines. Chakauya and Tongoona (2008) found that out of the ten primers screened for the ability to amplify and detect polymorphism among pearl millet landraces, four did not produce amplification products, four were monomorphic (PSMP2019, PSMP2059, PSMP2056, PSMP2069), while two primers (PSMP2008, PSMP2013) were polymorphic with 2 -4 bands ranging between $150-220$ base pairs. The two polymorphic primers were used for cluster analysis.

In previous studies, Stich et al. (2010) observed more number of alleles per locus, while analyzing diversity in germplasm lines. Similarly, Kapila et al. (2008) found an average of 6.26 alleles per locus, in a set of maintainer lines. In this study, lower number of alleles per locus observed, could be explained that we have used agarose gel, which has lower resolution power in comparison to polyacrylamide gel used in these studies. The level of polymorphism displayed by the SSR markers was considerably high in the present analysis, as showed by the high mean PIC value (0.51). The PIC values of SSR loci as genetic markers are also usually directly correlated to the type and number of repeats present. Kapila et al. (2008) also observed similar degree of SSR polymorphism, who recorded mean PIC values of 0.58 . Mean PIC varied across the linkage groups in pearl millet i.e. $0.44,0.55,0.67,0.57,0.66$,

Table 4. Distribution of forty nine pearl millet inbreds in different clusters based on SSR markers.

\begin{tabular}{lll}
\hline \multicolumn{1}{c}{ Inbreds } & Number of inbreds \\
\hline Cluster 1 & HSGR-01, HSGR-16, HSGR-12, HSGR-18, HSGR-21, HSGR-32, HSGR-37, HSGR-03, & 10 \\
& HSGR-08, HSGR-14 & \\
Cluster 2 & HSGR-13, HSGR-27, HSGR-33, HSGR-28, HSGR-44, HSGR-15 & 6 \\
Cluster 3 & HSGR-02, HSGR-19, HSGR-35, HSGR-06, HSGR-17, HSGR-20, HSGR-22, HSGR-43, & 9 \\
& H77/29-2 & 4 \\
Cluster 4 & HSGR-04, HSGR-09, HSGR-10, HSGR-42 & 6 \\
Cluster 5 & HSGR-24, HSGR-30, HSGR-40, HSGR-26,HSGR-34, HSGR-38 & 6 \\
Cluster 6 & HSGR-23, HSGR-36, H77/833-2-202,H77/833-2, HBL-11, ICMR-01004 & 5 \\
Cluster 7 & HSGR-07, HSGR-41, HSGR-25, HSGR-29, HSGR-31 & 5 \\
Cluster 8 & HSGR-05, HSGR-39, HSGR-11 & 3 \\
\hline
\end{tabular}


0.49 and 0.43 for LG1, LG2, LG3, LG4, LG5, LG6 and LG7, respectively. A number of reports are available in cereals that show different contributions of chromosomes/linkage groups to molecular diversity (Kleinhofs et al., 1993, Boyko et al., 1999, Huang et al., 2002, Ni et al., 2002). In pearl millet, LG 6 having the lowest number (1) of polymorphic SSR markers and low PIC (0.49) were observed in present study. Thus, the presence of some important genes involved in domestication might be responsible for conserved nature of LG 6 in pearl millet (Poncet et al., 2000).

Genetic diversity analysis: To understand the genetic relationships among the 49 inbreds, we clustered them into different groups by using WARD's minimum variance method (Fig. 3). On the basis of this method, 49 inbreds were separated into 8 major groups. Further, eight clusters grouped all the forty nine inbreds in such a way that inbreds within each cluster had high similarity than those in other clusters (Table 4). Cluster pattern revealed that, cluster1 was the largest consisting of 10 inbreds. This way followed by cluster3 (9 inbreds), cluster2 (6 inbreds), cluster5 (6 inbreds), cluster6 (6 inbreds), cluster7 (5 inbreds) and cluster4 (4 inbreds) and cluster8 (3 inbreds). Four inbreds, H77/833-2-202, H77/833-2, HBL-11 and ICMR-01004 were used as check lines in present study, grouped under same cluster (cluster6). This indicates that these genotypes might have an ancestral relationship.

The clustering pattern was not completely based on definite character. The set of primers used were not able to group all the genotypes into exactly similar phenotypically intended categories. This may be due to the fact that the numbers of SSR primers under study were not sufficient to represent all morphoagronomical characters.

Since pearl millet is highly cross-pollinated crop, hybrid development for commercial exploitation is a viable option. Depending upon the diversity available among inbred lines for trait of interest, these inbreds could effectively be utilized as parents through their conversion into male sterile and fertility restorer lines for developing commercial productive hybrids in pearl millet. Another possibility could be to develop openpollinated population using inbreds from different clusters and their subsequent genetic improvement through recurrent selection to concentrate desirable genes in the population which can further be used for developing improved inbred lines for targeted traits.

\section{Conclusion}

The study concludes that in case of pearl millet, the value of Polymorphic information content (PIC) ranged from 0.14 to 0.87 with an average of 0.518 specified the substantial efficiency of markers. Out of 70 primers, 29 primers showed higher polymorphism among the inbred lines. The study has provided an imminent of the inter-relationship among the geno- types and places of interest the genetic distance by SSR markers to competently reveal the genetic interrelationship among the genotypes. The study provides the basis for future pearl millet crop variety identification, maintenance, and management.

\section{ACKNOWLEDGEMENTS}

I would like to extend my gratitude to ICAR for providing junior research fellowship for the completion of master's degree.

\section{REFERENCES}

Anderson, J.A., Churchill, G.A., Autrique, J.E., Sorrells, M.E. and Tanksley, S.D. (1993). Optimizing parental selection for genetic linkage maps. Genome,36:181-186.

Anonymous (2014). Project Coordinator Review (AICPMIP) Jodhpur (Rajasthan). P.3.

Boyko, E. V., Gill, K. S., Mickelson-Young, L., Nasuda, S., Raupp, W. J., Ziegle, J. N., Singh, S., Hassawi, D. S., Fritz, A. K., Namuth, D., Lapitan, N. L. V. and Gill, B. S. (1999). A high density linkage map of Aegilops tauschii, the D-genome progenitor of bread wheat. Theoretical and Applied Genetics, 99:16-26

Chakauya, E. and Tongoona, P. (2008). Analysis of genetic relationships of pearl millet landraces from Zimbabwe, using microsatellites. International Journal of Plant Breeding and Genetics, 2:1-7

Ejeta, G., Hassen, M.M. and Mertz, E.T. (1987). In vitro digestibility and amino acid composition of pearl millet (Pennisetum typhoides) and other cereals. Proceedings of the National Academy of Sciences (USA), 84:60166019

Hill, G.M. and Hanna, W.W. (1990). Nutritive characteristics of pearl millet grain in beef cattle diets. Journal of Animal Sciences, 68:2061-2066.

Huang, X. Q., Borner, A., Roder, M.S. and Ganal, M.W. (2002). Assessing genetic diversity of wheat germplasm using microsatellite markers. Theoretical and Applied Genetics, 105: 699-707

Kannan, B., Senapathy, S., Raj, A.G.B., Chandra, S., Muthiah, A., Dhanapal, A.P. and Hash, C.H. (2014). Association Analysis of SSR Markers with Phenology, Grain, and Stover-Yield Related Traits in Pearl Millet. The Scientific World Journal. (http://dx.doi.org/ 10.1155/2014/562327)

Kapila, R.K., Yadav, R.S., Plaha, P., Rai, K.N., Yadav, O.P., Hash, C.T. and Howarth, C.J. (2008). Genetic diversity among pearl millet maintainers using microsatellite markers. Plant Breeding, 127: 33-37

Kleinhofs, A., Kilian, A., Saghai Maroof, M. A., Biyashev, R. M., Hayes, P., Chen, F. Q., Lapitan, N., Fenwick, A., Blake, T. K., Kanazin, V., Ananiev, E., Dahleen, L., Kudrna, D., Bollinger, J., Knapp, S. J., Liu, B., Sorrells, M., Heun, M., Franckowiak, J. D., Hoffman, D., Skadsen, R. and Steffenson, B. J. (1993). A molecular, isozyme and morphological map of the barley genome. Theoretical and Applied Genetics, 86:705-712

Liu, C.J., Witcombe ,J.R., Hash, C.T., Busso, C.S., Pittaway, T.S., Nash, M. and Gale, M.D. (1994). Witcombe JR, Duncan RR (eds): Use of molecular marker in sorghum and pearl millet breeding for developing countries. Oversease Development Administration: London U.K. 
pp. 57-69.

Murray, M.G. and Thompson, W.F. (1980). Rapid isolation of high molecular weight plant DNA. Nucleic Acids Research, 8:4321- 4325

Ni, J., Colowit, P. M. and Mackill, D. J. (2002). Evaluation of genetic diversity in rice subspecies using microsatellite markers. Crop Science, 42: 601-607

Poncet, V., Lamy, F., Devos, K. M., Gale, M. D., Sarr, A. and Robert, T. (2000). Genetic control of domestication traits in pearl millet. Theoretical and Applied Genetics, 100:147-159.

Saghai-Maroof, M.A., Soliman, K.M., Jorgensen, R.A. and Allad, R.W.(1984). Ribosomal DNA spacer length polymorphism in barley: Mendelian inheritance, Chromosomal location and Population dynamics. Proceedings of National Academy of Sciences, U.S.A., 81:8014-8019
Singh, A.K., Rana, M.K., Singh, S., Kumar, S., Durgesh, K. and Arya, L. (2013). Assesment of genetic diversity among pearl millet cultivars using SSR markers. Range Management and Agroforestry, 34:77-81

Stich, B., Haussmann, B.I.G. and Pasam, R. (2010). Patterns of molecular and phenotypic diversity in pearl millet from West and Central Africa and their relation to geographical and environmental parameters. BMC Plant Biology, 10:216

Sumathi, P. and Vinodhana, N.K. (2014). Estimation of Genetic relatedness among the germplasm accessions of Pearl millet. Plant Gene and Trait., 5:1-5

Weir, B.S. and Buckleton, J.S. (1996). Statistical issues in DNA profiling. Advances in Haemogenetics, 6:457-464

Xu, Y., Shimoro, X. and Hofstra, H. (1994). Plant DNA isolation protocol. Nucleic Acids Research, 22: 23992403 